Y. TAKEI

KODAI MATH. J.

15 (1992), 445-457

\title{
ON ALGEBRAICITY OF VECTOR VALUED SIEGEL MODULAR FORMS
}

\author{
BY YOSHINORI TAKEI
}

\section{Introduction.}

Let $n$ be a positive integer and let $k, l \geqq 0$ be integers. Let $S y m^{l}$ be the natural representation of $G L(n, \boldsymbol{C})$ on $S y m^{l}\left(\boldsymbol{C}^{n}\right)$, the $l$-th symmetric tensor product of the vector space $\boldsymbol{C}^{n}$.

A $\operatorname{Sym}^{l}\left(\boldsymbol{C}^{n}\right)$-valued holomorphic function $f$ on the Siegel upper half space of degree $n$ is called a Siegel modular form of degree $n$ and type $\operatorname{det}^{k} \otimes S y m^{l}$ when $f$ satifies certain automorphic condition with respect to the action of the integral symplectic group of size $2 \mathrm{n}$ through the representation $\operatorname{det}^{k} \otimes S y m^{l}$.

Let $M_{k, l}^{n}$ be the $\boldsymbol{C}$-vector space of Siegel modular forms of degree $n$ and type $\operatorname{det}^{k} \otimes S y m^{l}$. Let $S_{k, l}^{n}$ be the subspace of $M_{k, l}^{n}$ consisting of cuspforms. Precise definitions of them are in $\S 1$ below.

The purpose of this paper is to prove several algebraic results on Fourier coefficients of $f \in M_{k, l}^{n}$ described as follows:

RESULTS. (Precise statements are in $\xi 2$.)

Suppose that $k, l$ are even and $k \geqq 2 n+2$.

(1) $S_{k, l}^{n}$ has a basis consisting of forms whose Fourier coefficients lie in $\operatorname{Sym}^{l}\left(\boldsymbol{Q}^{n}\right)$.

(2) Let $f \in S_{k, l}^{n}$ be an eigenforms (i.e. a non-zero eigenfunction of the Hecke algebra) and let $\boldsymbol{Q}(f)$ be the extension field of $\boldsymbol{Q}$ generated by the eigenvalues on $f$ of the Hecke algebra over $\boldsymbol{Q}$. Then $\boldsymbol{Q}(f)$ is a totally real number field and the degree of extension does not exceed $S_{k, l}^{n}$.

(3) $S_{k, l}^{n}$ has an orthogonal basis consisting of eigenforms such that the Fourier coefficients of each element $f$ lie in $\operatorname{Sym}^{l}\left(\boldsymbol{Q}(f)^{n}\right)$.

(4) Let $m$ be a integer with $m \geqq n$ and $k>m+n+1$, let []$_{n}^{m}: S_{k, l}^{n} \rightarrow M_{k, l}^{m}$ be the Eisenstein lifting. Let $f \in S_{k, l}^{n}$ be an eigenform winose Fourier coefficients lie in $\operatorname{Sym}^{l}\left(\boldsymbol{Q}(f)^{n}\right)$. Then, $[f]_{n}^{m}$ has Fourier coefficients in $\operatorname{Sym}^{l}\left(\boldsymbol{Q}(f)^{m}\right)$.

For the case $l=0$, i.e. $\operatorname{Sym}^{0}\left(\boldsymbol{C}^{n}\right)=\boldsymbol{C}$-valued case, above results are proved by several authors. The assertion (2) is due to Kurokawa [6]. In [5], Garrett

Received March 9, 1992. 
showed the "Pullback Formula" which reduces problems on Siegel modular forms to smaller degree ones, and using this formula he showed (a similitude of) (4). Böcherer [4] showed (3), by effective use of the pullback formula. In [7], Mizumoto gave a way to prove (3), (4) as well as (1) simulatenously, also using the pullback formula.

In the paper [2] of Böcherer-Satoh-Yamazaki, they have obtained the pullback formula for the case $l \in \mathbf{2} \boldsymbol{Z}>0$, which enables us to apply the above proofs for the case $l=0$ to the case $l \in \mathbf{Z} \boldsymbol{Z}>0$ without essential change. A brief description of the pullback formula is given in $\S 3$, where we shall also a connection between Fourier coefficients of eigen cuspforms and the partial Fourier expansion of pullback of Siegel's Eisenstein series.

The stated results shall be proved in $\S 4$.

The author would like to express his gratitude to Professor S. Mizumoto for helpful advices, and to Professor T. Harase for constant encouragement.

\section{Notations and definitions.}

Let $n$ be a positive integer and $k, l$ be positive even integers. Let $\boldsymbol{x}:=$ $\left(x_{1}, \cdots, x_{n}\right)$ be a row vector with $x_{1}, \cdots, x_{n}$ being indeterminantes. We define a $\boldsymbol{C}$-vector space

$$
V:=\boldsymbol{C} x_{1} \oplus \cdots \oplus \boldsymbol{C} x_{n}
$$

and a Hermitian inner product on $V$ by

$$
\left(\sum_{\imath=1}^{n} a_{\imath} x_{\imath}, \sum_{i=1}^{n} b_{i} x_{\imath}\right):=\sum_{i=1}^{n} a_{i} \bar{b}_{\imath}
$$

where $a_{\imath}, b_{i} \in \boldsymbol{C}(1 \leqq i \leqq n)$ and $\bar{b}_{\imath}$ denotes the complex conjugate of $b_{i}$. Put $V^{(l)}:=\operatorname{Sym}^{l}(V)$, the $l$-th symmetric tensor product of $V$, which is identified with $\boldsymbol{C}\left[x_{1}, \cdots, x_{n}\right]_{(l)}$, the $\boldsymbol{C}$-vector space of homogeneous polynomials in $x_{1}, \cdots$, $x_{n}$ of degree $l$. The inner product (1.1) induces an inner product on $V^{(l)}$ by

$$
\left(\alpha_{1} \cdots \alpha_{l}, \beta_{1} \cdots \beta_{l}\right):=\frac{1}{l !} \sum_{\sigma \in \subseteq_{l}} \prod_{j=1}^{l}\left(\alpha_{\sigma(j)}, \beta_{j}\right)
$$

where $\alpha_{\jmath}, \beta_{j} \in V$, - denotes the symmetric tensor product and $\mathfrak{S}_{l}$ denotes the symmetric group of degree $l$.

Let $\rho=\rho_{k, l}^{n}$ be the representation

$$
\operatorname{det}^{k} \otimes S y m^{l}: G L(n, C) \longrightarrow G L\left(V^{(l)}\right) .
$$

Let $\mathfrak{S}_{n}$ be Siegel upper half space of degree $n$, and $\Gamma_{n}:=S p(n, \boldsymbol{Z})$ be the group of integral symplectic group of size $2 n$.

For a function $f: \mathfrak{S}_{n} \rightarrow V^{(l)}$ and $M \in S p(n, \boldsymbol{R})$, put

$$
\left.f\right|_{k, l} ^{n} M(Z):=\rho(C Z+D)^{-1} f(M\langle Z\rangle)
$$


with

$$
M\langle Z\rangle:=(A Z+B)(C Z+D)^{-1}
$$

for $Z \in \mathfrak{S}_{n}$ and $M\left(\begin{array}{ll}A & B \\ C & D\end{array}\right)$.

The $\boldsymbol{C}$-vector space of $V^{(l)}$-valued Siegel modular forms of degree $n$ and type $k, l$ with respect to $\Gamma_{n}$ is defined by

(1.3) $M_{k}^{n}{ }_{l}\left(V^{(l)}\right):=\left\{f: \mathfrak{S}_{n} \rightarrow V^{(l)} \mid f\right.$ is holomorphic on $\mathfrak{Y}_{n}$ (and at the cusps if $n=1)$, and $\left.f\right|_{k, l} ^{n} M=f$ for all $\left.M \in \Gamma_{n}\right\}$,

and the space of cuspforms by

$$
\begin{aligned}
S_{k, l}^{n}\left(V^{(l)}\right):=\left\{f \in M_{k, l}^{n}\left(V^{(l)}\right) \mid\right. \\
\left.\quad \lim _{\lambda \rightarrow \infty} f\left(\begin{array}{cc}
z & 0 \\
0 & \sqrt{-1 \lambda}
\end{array}\right)=0 \text { for all } z \in \mathfrak{S}_{n-1}\right\} .
\end{aligned}
$$

In the notations (1.3) and (1.4), we omit $\left(V^{(l)}\right)$ whenever $V$ is obvious. For $l=0, M_{k, 0}^{n}\left(V^{(0)}\right)=M_{k, 0}^{n}(\boldsymbol{C})$ is the space of Siegel modular forms of weight $k$.

Each $f \in M_{k, l}^{n}\left(V^{(l)}\right)$ has a Fourier expansion of the following type:

$$
f(Z)=\sum_{R \geq 0} a(R ; f) \mathbf{e}(R Z)\left(a(R ; f) \in V^{(l)}, Z \in \mathfrak{H}_{n}\right)
$$

where $\mathbf{e}(\cdot):=\exp 2 \pi \sqrt{-1} \operatorname{trace}(\cdot)$, and $R$ runs through symmetric, semiintegral, semipositive matrices of size $n$ (We denote such $R$ by " $R \geqq 0$ " or by " $R^{(n)} \geqq 0$ "). If $f$ is a cuspform, then $a(R ; f) \neq 0$ only for $R>0$. Throughout this paper, $a(R ; f)$ denotes the Fourier coefficient of $f$ at $R$.

Let $\operatorname{Aut}(\boldsymbol{C})$ be the group of all field automorphisms of $\boldsymbol{C}$. For $\tau \in \operatorname{Aut}(\boldsymbol{C})$ and a function $f(Z)=\sum_{R \geqq 0} a(R ; f) \mathbf{e}(R Z)$, set

$$
f^{\tau}(Z):=\sum_{R \geq 0} a(R ; f)^{\tau} \mathbf{e}(R Z) .
$$

Let $K$ be any subfield of $\boldsymbol{C}$. Put

$$
\begin{aligned}
& V_{K}:=K x_{1} \oplus \cdots \oplus K x_{n} \\
& M_{k, l}^{n}\left(V^{(l)}\right)_{K}:=\left\{f \in M_{k, l}^{n} \mid a(R ; f) \in V_{K}^{(l)} \text { for all } R^{(n)} \geqq 0\right\}
\end{aligned}
$$

and for any subset $X$ of $M_{k, l}^{n}\left(V^{(l)}\right)$, set

$$
X_{K}:=X \cap M_{k, l}^{n}\left(V^{(l)}\right)_{K} .
$$

Let $r \leqq n$ and put $V_{r}:=\boldsymbol{C} x_{n-r+1} \oplus \cdots \oplus \boldsymbol{C} x_{n}$.

For $1 \leqq r \leqq n$ with even $k>n+r+1$, the Langlands-Klingen type Eisenstein series $[f]_{r}^{n} \in M_{k, l}^{n}\left(V^{(l)}\right)$ is attached to $f \in S_{k, l}^{r}\left(V_{r}^{(l)}\right)$ by

$$
[f]_{r}^{n}(Z)=\sum_{M \in P_{n, r} \backslash \Gamma_{n}} \rho(C Z+D)^{-1} f\left(M\langle Z\rangle^{*}\right)
$$


where $Z \in \mathfrak{S}_{n}, M=\left(\begin{array}{cc}A & B \\ C & D\end{array}\right), M\langle Z\rangle^{*}$ denotes the lower-right $r \times r$ block of $M\langle Z\rangle$, and $P_{n, r}=\left\{\left(\begin{array}{cc}* & * \\ 0^{(n-r, n+r)} & *\end{array}\right) \in \Gamma_{n}\right\}$ which is a subgroup of $\Gamma_{n}$. The linear map []$_{r}^{n}: S_{k, l}^{r} \rightarrow M_{k, l}^{n}$ is called the Eisenstein lifting. We define []$_{n}^{n}$ as the identity map on $S_{k, l}^{n}$. When $l=0$, the Eisenstein lifting is also defined for $r=0$. In this case, we understand that $M_{k, 0}^{0}(\boldsymbol{C})=S_{k, 0}^{0}(\boldsymbol{C})=\boldsymbol{C}$, and the Eisenstein lift of $f=1$

$$
E_{k}^{n}(Z):=[1]_{0}^{n}(Z)=\sum_{M \in P_{n, 0} \backslash \Gamma_{n}} \operatorname{det}(C Z+D)^{-k}
$$

is Siegel's original Eisentein series [8].

For $f, g \in M_{k, l}^{n}$ (at least one in $S_{k, l}^{n}$ ), their Pertersson inner product $(f, g)$ is defined by:

$$
(f, g):=\int_{\Gamma_{n} \backslash \mathfrak{\xi}_{n}}(\rho(\sqrt{Y}) f(Z), \rho(\sqrt{Y}) g(Z))(\operatorname{det} Y)^{-n-1} d X d Y
$$

with $Z=X+\sqrt{-1} Y, X, Y$ real and (, ) in the right-hand side is the inner product (1.2) defined on $V^{(l)}$.

We note that if $r<n$, then

$$
\left(f,[\phi]_{r}^{n}\right)=0 \quad \text { for all } f \in S_{k, l}^{n} \text { and } \phi \in S_{k, l}^{r} .
$$

Let $L_{C}^{(n)}\left(\right.$ resp. $\left.L_{\boldsymbol{Q}}^{(n)}\right)$ be the abstract Hecke algebra of degree $n$ over $\boldsymbol{C}$ (resp. $\boldsymbol{Q})$ and let

$$
t: L_{\boldsymbol{C}}^{(n)} \longrightarrow \operatorname{End}_{C}\left(S_{k, l}^{n}\right) .
$$

be the $\boldsymbol{C}$-algebra homomorphism defined as in [1].

We put $\boldsymbol{T}_{C}:=t\left(L_{\boldsymbol{c}}^{(n)}\right)$ and $\boldsymbol{T}_{\boldsymbol{Q}}:=t\left(L_{\boldsymbol{Q}}^{(n)}\right)$. Let $f \neq 0 \in S_{k, l}^{n}$ be a common eigenfunction to all $T \in \boldsymbol{T}_{C}$ (such $f$ is called an eigenform), and for each $T$, let $\lambda(T) \in \boldsymbol{C}$ be the eigenvalue on $f:$

$$
T f=\lambda(T) f \quad \text { for all } T \in \boldsymbol{T}_{C} .
$$

Then $\lambda$ is a $\boldsymbol{C}$-algebra homomorphism $\lambda: \boldsymbol{T}_{\boldsymbol{C}} \rightarrow \boldsymbol{C}$ and each element of $\widehat{\boldsymbol{T}_{\boldsymbol{C}}}:=$ Hom $_{C \text {-alg }}\left(\boldsymbol{T}_{\boldsymbol{C}}, \boldsymbol{C}\right)$ is obtained in this way.

For each $\lambda \in \widehat{\boldsymbol{T}_{\boldsymbol{C}}}$, put

$$
S_{k, l}^{n}(\lambda):=\left\{f \in S_{k l}^{n} \mid T f=\lambda(T) f \text { for all } T \in \boldsymbol{T}_{c}\right\} .
$$

Then the space of cuspforms decomposes into eigenspaces:

$$
S_{k, l}^{n}=\underset{\lambda \in \widehat{T_{C}}}{\bigoplus} S_{k, l}^{n}(\lambda) \text {. }
$$

We note that for any $f_{1} \in S_{k, l}^{n}\left(\lambda_{1}\right)$ and $f_{2} \in S_{k, l}^{n}\left(\lambda_{2}\right),\left(f_{1}, f_{2}\right)=0$ if $\lambda_{1} \neq \lambda_{2}$.

For each $\lambda \in \widehat{\boldsymbol{T}_{\boldsymbol{C}}}$, define an extension field of $\boldsymbol{Q}$ by 


$$
\boldsymbol{Q}(\lambda):=\boldsymbol{Q}\left(\lambda(T) \mid T \in \boldsymbol{T}_{\boldsymbol{Q}}\right),
$$

and for $f \in S_{k, l}^{n}(\lambda)$ put $\boldsymbol{Q}(f):=\boldsymbol{Q}(\lambda)$.

\section{Statement of the Theorems.}

THEOREM 1. Let $q \geqq 1$ be an integer, let $k, l \geqq 0$ be even integers satisfying

$$
k \geqq 2 q+2 \text {. }
$$

Then, the following holds.

$$
S_{k, l}^{q}=S_{k, l_{Q}}^{q} \otimes_{Q} C .
$$

In partıcular, Aut $(\boldsymbol{C})$ acts on $S_{k, l}^{q}$ by $f \mapsto f^{\tau}$ in the notation (1.6).

(2) Let $\lambda \in \widehat{\boldsymbol{T}_{C}}$ and $f \neq 0 \in S_{k}^{q} l(\lambda)_{Q(\lambda)}$.

(i) $\boldsymbol{Q}(\lambda)$ is a totally real finite extention of $\boldsymbol{Q}$ with

$$
[\boldsymbol{Q}(\lambda): \boldsymbol{Q}] \leqq \operatorname{dim}_{C} S_{k, l}^{q} \text {. }
$$

(ii) Let $c(f)$ be the constant of (3.5) below. Then,

$$
\left(\frac{c(f)}{(f, f)}\right)^{\tau}=\frac{c\left(f^{\tau}\right)}{\left(f^{\tau}, f^{\tau}\right)} \quad \text { for all } \quad \tau \in \operatorname{Aut}(\boldsymbol{C}) .
$$

(iii) Let $m:=\operatorname{dim}_{C} S_{k, l}^{q}(\lambda)$. There exists an orthogonal basis $\left\{f_{j}\right\}_{j=1}^{m}$ of $S_{k, l}^{q}(\lambda)$ such that

$$
f_{1}=f \quad \text { and } \quad f_{j} \in S_{k, l}^{q}(\lambda)_{Q(\lambda)}(1 \leqq \jmath \leqq m) .
$$

THEOREM 2. Let $p \geqq q \geqq 1$ be integers, let $k, l \geqq 0$ be even integers satisfying

$$
k>p+q+1 \text {. }
$$

Let $\lambda \in \widehat{\boldsymbol{T}}_{\boldsymbol{C}}$ and $f \neq 0 \in S_{k, l}^{q}(\lambda)_{\boldsymbol{Q}}(\lambda)$. Then,

$$
\left([f]_{q}^{p}\right)^{\tau}=\left[f^{\tau}\right]_{q}^{p} \quad \text { for all } \quad \tau \in \operatorname{Aut}(\boldsymbol{C}) .
$$

\section{Differential operators and the Pullback formula.}

The first part of this section is a brief description of the "Pullback Formula" of Böcherer-Satoh-Yamazaki [2].

Let $p, q \geqq 1$ be integers. Put

$$
\begin{aligned}
& V_{\boldsymbol{x}}:=\boldsymbol{C} x_{1} \oplus \cdots \oplus \boldsymbol{C} x_{p}, \quad \boldsymbol{x}:=\left(x_{1}, \cdots, x_{p}\right) \\
& V_{\boldsymbol{y}}:=\boldsymbol{C} y_{1} \oplus \cdots \oplus \boldsymbol{C} y_{q}, \quad \boldsymbol{y}:=\left(y_{1}, \cdots, y_{q}\right) .
\end{aligned}
$$

and for $r \leqq \min (p, q)$, put 


$$
\begin{aligned}
& V_{\boldsymbol{x}, r}:=\boldsymbol{C} x_{p-r+1} \oplus \cdots \oplus \boldsymbol{C} x_{p}, \\
& V_{\boldsymbol{y}, r}:=\boldsymbol{C} y_{q-r+1} \oplus \cdots \oplus \boldsymbol{C} y_{q} .
\end{aligned}
$$

and define an isomorphism $\sigma: V_{\boldsymbol{x}, r} \rightarrow V_{\boldsymbol{y}, r}$ by $\sigma\left(x_{p-\jmath}\right)=y_{q-\jmath}(\jmath<\min (p, q))$.

Let $3=\left(\mathfrak{Z}_{i \jmath}\right)_{1 \leqq \imath, \jmath \leqq p+q}$ be a variable on $\mathfrak{H}_{p+q}$ and

$$
\left(\frac{\partial}{\partial 3}\right)=\left(\frac{1+\delta_{i \jmath}}{2} \frac{\partial}{\partial 3_{i \jmath}}\right)_{1 \leqq i, \jmath \leqq p+q} .
$$

For a holomorphic function $f: \mathfrak{S}_{p+q} \rightarrow\left(V_{\boldsymbol{x}} \oplus V_{\boldsymbol{y}}\right)^{(l)}$, we define the operators

$$
\begin{aligned}
& D f:=\frac{1}{2 \pi \sqrt{-1}}\left(\begin{array}{ll}
\boldsymbol{x} & \boldsymbol{y}
\end{array}\right)\left(\frac{\partial}{\partial \mathfrak{3}}\right) f\left(\begin{array}{l}
{ }^{t} \boldsymbol{x} \\
{ }^{t} \boldsymbol{y}
\end{array}\right), \\
& D_{\uparrow} f:=-\frac{1}{2 \pi \sqrt{-1}}\left(\begin{array}{ll}
\boldsymbol{x} & 0
\end{array}\right)\left(\frac{\partial}{\partial 3}\right) f\left(\begin{array}{c}
{ }^{t} \boldsymbol{x} \\
0
\end{array}\right), \\
& D_{\downarrow} f:=\frac{1}{2 \pi \sqrt{-1}}\left(\begin{array}{ll}
0 & \boldsymbol{y}
\end{array}\right)\left(\frac{\partial}{\partial \boldsymbol{3}}\right) f\left(\begin{array}{c}
0 \\
{ }^{t} \boldsymbol{y}
\end{array}\right) \text {. }
\end{aligned}
$$

Let $d$ be the diagonal embedding

$$
\begin{gathered}
d: \mathfrak{H}_{p} \times \mathfrak{H}_{q} \longrightarrow \mathfrak{H}_{p+q} \\
(Z, W) \longmapsto\left(\begin{array}{rr}
Z & 0 \\
0 & W
\end{array}\right)
\end{gathered}
$$

and let $d^{*}$ be the pullback of $d$.

The differential operator $L^{(l)}$ is defined in [2] as follows:

$$
\begin{aligned}
L^{(l)}= & d^{*} \frac{1}{k^{[l]}} \\
& \times \sum_{0 \leq 2 \nu \leq l} \frac{1}{\nu !(l-2 \nu) !(2-k-l)^{[\nu]}}\left(D_{\uparrow} D_{\downarrow}\right)^{2 \nu}\left(D-D_{\uparrow}-D_{\downarrow}\right)^{l-2 \nu},
\end{aligned}
$$

where

$$
a^{[b]}:= \begin{cases}\frac{(a+b-1) !}{(a-1) !} & \text { for } a>0 \\ 1 & \text { otherwise }\end{cases}
$$

for integers $a$ and $b$.

This defines a linear map

$$
L^{(l)}: M_{k, 0}^{p+q}(\boldsymbol{C}) \longrightarrow M_{k, l}^{p}\left(V_{\boldsymbol{x}}{ }^{(l)}\right) \otimes M_{k, l}^{q}\left(V_{\boldsymbol{y}}{ }^{(l)}\right) .
$$

ThEOREM A [2, Prop. 4.4]. Let $p, q \geqq 1$ be integers and $k, l \geqq 2$ be even integers satısfying $k>p+q+1$. For each $1 \leqq r \leqq \min (p, q)$, let $d(r):=\operatorname{dim}_{C}$ $S_{k, l}^{r}\left(V_{y, r}{ }^{(l)}\right)$ and $\left\{f_{j, r}\right\}_{j=1}^{d(r)}$ an orthonormal basis of $S_{k, l}^{r}\left(V_{y, r}{ }^{(l)}\right)$ consisting of 

eigenforms.

Let $E_{k}^{p+q} \in M_{k, 0}^{p+q}(\boldsymbol{C})$ be Siegel's Eisenstern series (1.10) of degree $p+q$ and weight $k$. Let $\alpha_{k, l}$ and $C_{k, l, r}$ be the constants

$$
\begin{aligned}
\alpha_{k, l}= & \left(-\frac{1}{2 \pi \sqrt{-1}}\right)^{l} \frac{(2 k-2)^{[l]}}{l !(k-1)^{[l]}}, \\
C_{k, l, r}= & 2^{r(r-k+1)-l+1} \sqrt{-1^{r k+l}} \frac{\pi^{r(r+1) / 2}}{k+l-1} \\
& \times \prod_{u=1}^{r-1} \frac{\Gamma(2 k-2 r+2 u-1)(2 k-r+u-2)^{[l]}}{(k-r-1+u) \Gamma(2 k+u+l-r-1)} .
\end{aligned}
$$

For an eigenform $f \in S_{k, l}^{r}\left(V_{\boldsymbol{y}, r}{ }^{(l)}\right)$, put

$$
\begin{aligned}
\theta f(Z) & :=\overline{f(-\bar{Z})}, \\
\Lambda(f) & :=\left(\zeta(k)^{-1} \prod_{\imath=1}^{r} \zeta(2 k-2 i)^{-1}\right) L(k-r, f, \mathbf{S} t),
\end{aligned}
$$

where $\zeta$ denotes Riemann zeta function and $L(*, f, \boldsymbol{S} t)$ denotes the standard $L$ function attached to $f$, respectively.

Then, following equation holds

$$
\begin{aligned}
L^{(l)} E_{k}^{p+q}(Z, W)= & \alpha_{k, l} \sum_{r=1}^{m \imath n(p, q)} C_{k, l, r} \sum_{j=1}^{d(r)} \\
& \Lambda\left(f_{\jmath, r}\right)\left[\theta \sigma^{-1} f_{\jmath, r}\right]_{r}^{p}(Z)\left[f_{\jmath, r}\right]_{r}^{q}(W) .
\end{aligned}
$$

In the rest of this section, we study a connection between Fourier coeff. cients of eigenforms and the partial Fourier expansion of $L^{(l)} E_{k}^{p+q}$.

Let $p, q, k$ be as in the assumption of Theorem $\mathrm{A}$ and suppose also $p \geqq q$. Let $R=R^{(p)} \geqq 0$ be a symmetric, semiintegral, semipositive matrix of size $p$. Let $X_{p}=\left\{\xi=\Pi_{i=1}^{p} x_{\imath}^{\alpha_{i}} \mid a_{i} \in \boldsymbol{Z} \geqq 0, \sum_{i} a_{i}=l\right\}$, which is an orthonormal basis of $\boldsymbol{C}$ vector space $V_{x}{ }^{(l)}$.

We attach a $V_{y}{ }^{(l)}$-valued modular form $g_{R, \xi}^{p, q} \in M_{k, l}^{q}\left(V_{y}{ }^{(l)}\right)$ for each $R \geqq 0$ and $\xi \in X_{p}$ through the partial Fourier expantion of $L^{(l)} E_{k}^{p+q}$ :

$$
L^{(l)} E_{k}^{p+q}(Z, W)=\sum_{R \geqq 0} \sum_{\xi \in X} g_{p}^{p, q}, \xi(W) \xi \mathbf{e}(R Z) .
$$

Since the Fourier coefficients of Siegel's Eisenstein series are rational, and $L^{(l)}$ preserves rationality of Fourier coefficients, we have

$$
g_{R, \xi}^{p, q} \in S_{k, l}^{q}\left(V_{\boldsymbol{y}}{ }^{(l)}\right)_{\boldsymbol{Q}} .
$$

For $F \in M_{k, l}^{p}\left(V_{\boldsymbol{x}}{ }^{(l)}\right)$ and $R=R^{(p)} \geqq 0$ and $\xi \in X_{p}$, let $a(R ; F ; \xi)$ denote the $\xi$ component of the Fourier coefficient $a(R ; F)$.

For each eigenform $f \in S_{k, l}^{q}\left(V_{y}{ }^{(l)}\right)$, put

$$
c(f):=\alpha_{k, l} C_{k, l, q} \Lambda(f) \text {. }
$$


We note that $c(f)$ is a nonzero constant depending only on $\lambda \in \widehat{\boldsymbol{T}_{\boldsymbol{C}}}$ such that $S_{k, l}^{q}\left(V_{\boldsymbol{y}}{ }^{(l)} ; \lambda\right) \ni f$. We occasionally write $c(f)$ as $c(\lambda)$ for such $\lambda$. By Theorem A, taking inner product of $f$ and $L^{(l)} E_{k}^{p+q}(-\bar{Z}, *)$ on $S_{k, l}^{q}\left(V_{\boldsymbol{y}}{ }^{(l)}\right)$, we obtain

$$
\left(f, g_{R, \xi}^{p, q}\right)=c(f) a\left(R ;\left[\sigma^{-1} f\right]_{q}^{p} ; \xi\right)\left(R^{(p)} \geqq 0, \xi \in X_{p}\right) .
$$

In the rest of the paper, we simply write $M_{k, l}^{q}\left(V_{\boldsymbol{y}}{ }^{(l)}\right)$ (resp. $\left.S_{k, l}^{q}\left(V_{\boldsymbol{y}}{ }^{(l)}\right)\right)$ as $M_{k, l}^{q}\left(\operatorname{resp} . S_{k, l}^{q}\right)$.

Let $h_{R, \xi}^{p, q}$ be the projection of $g_{R, \xi}^{p, q}$ to $S_{k, l}^{q}$. Then, for each eigenform $f \in$ $S_{k, l}^{q}$, we get

$$
\left(f, h_{R, \xi}^{p, q}\right)=c(f) a\left(R ;\left[\sigma^{-1} f\right]_{q}^{p} ; \xi\right)\left(R^{(p)} \geqq 0, \xi \in X_{p}\right) .
$$

In particular, when $p=q$,

$$
\left(f, h_{R, \xi}^{q, q}\right)=c(f) a\left(R ; \sigma^{-1} f ; \xi\right)\left(R^{(q)} \geqq 0, \xi \in X_{q}\right) .
$$

PROPOSITION. Let $q \geqq 1$ be an integer and $k, l \geqq 0$ be even integers satısfying

$$
k \geqq 2 q+2 \text {. }
$$

Then,

$$
S_{k, l}^{q}=\left\langle h_{R, \xi}^{q, q} \mid R^{(q)}>0, \xi \in X_{q}\right\rangle_{c},
$$

where \langle\rangle$_{c}$ means the $\boldsymbol{C}$-linear span.

Proof. Let $S$ be the space in the right-hand side of (3.10), and $S^{\perp}$ be its orthogonal complement in $S_{k, l}^{q}$. Let $f$ be any eigenform in $S_{k, l}^{q}$. By (3.8), $f \in$ $S^{\perp}$ if and only if $f=0$. Since $S_{k, l}^{q}$ has an orthogonal basis consisting of eigenforms, we see that $S^{\perp}=0$.

\section{Proof of Theorems.}

We shall prove Theorems 1,2 by similar way as in [7]. First, we introduce a condition on $(p, q)$.

Condition $C(p, q)$ :

$$
h_{R, \xi}^{p, q} \in S_{k, l_{Q}}^{q} \quad \text { for all } \quad R=R^{(p)} \geqq 0 \text { and } \xi \in X_{p} .
$$

We first show that Theorems 1,2 are valid for $(p, q)$ which satisfy $C(p, q)$ and $C(q, q)$.

We write the assertion of Theorem 1 for $q$ as $A(q)$ and the assertion of Theorem 2 for $(p, q)$ as $B(p, q)$.

Proposition 4.1. ( I ) Suppose $q \geqq 1$ satisfies $C(q, q)$. Then $A(q)$ holds.

(II) Suppose $p \geqq q \geqq 1$ satisfy $C(p, q)$ and $C(q, q)$. Then $B(p, q)$ holds.

Proof. (I) Suppose that $k \geqq 2 q+2$. From (3.10) and $C(q, q), A(q)$ (1) fol- 
ALGEBRAICITY OF VECTOR VALUED SIEGEL MODULAR FORMS

lows immediately. Next, we show $A(q)(2)(\mathrm{i})$ following [6]. There exists the action of $\operatorname{Aut}(\boldsymbol{C})$ on $\widehat{\boldsymbol{T}_{\boldsymbol{C}}}$ which is defined by

$$
\lambda^{\tau}(T):=\lambda(T)^{\tau} \quad\left(T \in \boldsymbol{T}_{Q}\right)
$$

with $\lambda \in \widehat{\boldsymbol{T}_{\boldsymbol{C}},} \in \operatorname{Aut}(\boldsymbol{C})$ and by

$$
\boldsymbol{T}_{C}=\boldsymbol{T}_{Q} \otimes_{Q} \boldsymbol{C} .
$$

By $A(q)(1)$ and similar argument to [6, Theorem 1], we have

$$
(T f)^{\imath}=T\left(f^{\imath}\right) \quad \text { for all } f \in S_{k, l}^{q}, T \in \boldsymbol{T}_{\boldsymbol{Q}}, \tau \in \operatorname{Aut}(\boldsymbol{C}) .
$$

In particular, for all $\tau \in \operatorname{Aut}(\boldsymbol{C})$ we have

$$
f^{\imath} \in S_{k, l}^{q}\left(\lambda^{2}\right) \quad \text { for } \quad f \in S_{k, l}^{q}(\lambda) \text { and } \tau \in \operatorname{Aut}(\boldsymbol{C})
$$

and

$$
\boldsymbol{Q}\left(\lambda^{\tau}\right)=\boldsymbol{Q}(\lambda)^{\tau} .
$$

Since Aut $(\boldsymbol{C})$ acts on $\widehat{\boldsymbol{T}_{\boldsymbol{C}}}$ whose cardinality $\leqq \operatorname{dim}_{C} S_{k, l}^{q}$, we get $[\boldsymbol{Q}(\lambda): \boldsymbol{Q}] \leqq$ $\operatorname{dim}_{C} S_{k, l}^{q}$. The field $\boldsymbol{Q}(\lambda)$ is totally real since all $T \in \boldsymbol{T}_{\boldsymbol{Q}}$ are Hermitian.

Next, we shall show $A(q)(2)$ (iii). Put $d=\operatorname{dim}_{C} S_{k, l}^{q}$. We choose $\left\{\left(R_{\imath}, \xi_{\imath}\right) \mid\right.$ $\left.R_{\imath}>0, \xi_{i} \in X_{q}, 1 \leqq i \leqq d\right\}$ so that

$$
\left\{h_{R_{i}, \xi_{i}}^{q, q} \mid i=1, \cdots, d\right\}
$$

is a $\boldsymbol{C}$-basis of $S_{k, l}^{q}$. We claim that this is also a $\boldsymbol{Q}$-basis of $S_{k, l_{\boldsymbol{Q}}}^{q}$. For any $h \in S_{k, l_{Q}}^{q}$, there exists unique $\left(\alpha_{1}, \cdots, \alpha_{d}\right) \in C^{d}$ such that

$$
h=\sum_{i=1}^{d} \alpha_{i} h_{R_{i}, \xi_{i}}^{q, q}
$$

Since $h, h_{R_{i}, \xi_{i}}^{q_{q}} \in S_{k, l_{Q}}^{q}$ by the assumption, we get

$$
h=\sum_{i=1}^{d} \alpha_{i}^{\tau} h_{R_{i}, \xi_{i}}^{q, q} \quad \text { for all } \quad \tau \in \operatorname{Aut}(\boldsymbol{C}),
$$

but by the uniqueness of $\left(\alpha_{1}, \cdots, \alpha_{d}\right)$, we get $\left(\alpha_{1}, \cdots, \alpha_{d}\right)^{\tau}=\left(\alpha_{1}, \cdots, \alpha_{d}\right)$ for all $\tau \in \operatorname{Aut}(\boldsymbol{C})$.

Hence, $\left(\alpha_{1}, \cdots, \alpha_{d}\right) \in \boldsymbol{Q}^{d}$, and we see that

$$
\left\{h_{R_{i}, \xi_{i}}^{q_{i},} \mid \imath=1, \cdots, d\right\}
$$

is a $\boldsymbol{Q}$-basis of $S_{k, l_{Q}}^{q}$.

For $T \in \boldsymbol{T}_{\boldsymbol{Q}}$ let $B(T) \in M_{d}(\boldsymbol{C})$ be the representation matrix of $T$ with respect to the basis (4.2). Since $S_{k_{, l_{\boldsymbol{Q}}}}$ is $\boldsymbol{T}_{\boldsymbol{Q}}$-stable, $B(T)$ lies in $M_{d}(\boldsymbol{Q})$.

For $\lambda \in \widehat{\boldsymbol{T}_{C}}$, put $m=m(\lambda):=\operatorname{dim}_{C} S_{k, l}^{q}(\lambda)$. Let $\left\{\boldsymbol{a}_{1}, \cdots, \boldsymbol{a}_{m}\right\}$ be column vectors in $\boldsymbol{C}^{d}$ which spans $\left\{\boldsymbol{a} \in \boldsymbol{C}^{d} \mid\left(B(T)-\lambda(T) 1_{d}\right) \boldsymbol{a}=0\right.$ for all $\left.T \in \boldsymbol{T}_{\boldsymbol{Q}}\right\}$. Since $B(T) \in$ $M_{d}(\boldsymbol{Q})$ and $\lambda(T) \in \boldsymbol{Q}(\lambda)$, we can take such $\left\{\boldsymbol{a}_{1}, \cdots, \boldsymbol{a}_{m}\right\}$ in $\boldsymbol{Q}(\lambda)^{d}$. Put 


$$
\phi_{\jmath}=\left(h_{R_{1}, \xi_{1}}^{q_{1},} \cdots h_{R_{d}, \xi_{d}}^{q_{1} q}\right) \boldsymbol{a}_{\jmath} \quad(1 \leqq j \leqq m) .
$$

Then, $\left\{\phi_{\jmath}\right\}_{j=1}^{m}$ is a $\boldsymbol{C}$-basis of $S_{k, l}^{q}(\lambda)$, which is also a $\boldsymbol{Q}(\lambda)$-basis of $S_{k, l}^{q}(\lambda)_{\boldsymbol{Q}(\lambda)}$.

For given $f \neq 0 \in S_{k, l}^{q}(\lambda)_{Q(\lambda)}$, we choose an index $\jmath_{0}$ so that $\left\{\phi_{j} \mid 1 \leqq \jmath \leqq m, j \neq\right.$ $\left.\jmath_{0}\right\} \cup\{f\}$ is a $\boldsymbol{Q}(\lambda)-\left(\right.$ resp. $\boldsymbol{C}$-) basis of $S_{k, l}^{q}(\lambda)_{\boldsymbol{Q}(\lambda)}$ (resp. $\left.S_{k, l}^{q}(\lambda)\right)$. Let $\jmath_{0}=m$ by changing order.

For any $\phi \in S_{k, l}^{q}(\lambda)_{Q(\lambda)}$, (3.8) implies

$$
\begin{aligned}
\left(\phi, \phi_{j}\right) & =\left(\left(\psi, h_{R_{1}, \xi_{1}}^{q_{q}}\right) \cdots\left(\psi, h_{R_{d}, \xi_{d}}^{q_{,} q}\right)\right) \boldsymbol{a}_{J} \\
& =c(\lambda)\left(a\left(R_{1} ; \sigma^{-1} \phi ; \xi_{1}\right) \cdots a\left(R_{d} ; \sigma^{-1} \phi ; \xi_{d}\right)\right) \boldsymbol{a}_{\jmath} \\
& \leqq c(\lambda) \cdot \boldsymbol{Q}(\lambda) \quad(1 \leqq \jmath \leqq m-1)
\end{aligned}
$$

and in particular,

$$
\frac{\left(\phi, \phi_{\jmath}\right)}{\left(\phi_{\jmath}, \phi_{j}\right)} \in \boldsymbol{Q}(\lambda) \quad\left(1 \leqq \jmath \leqq m-1, \phi \in\{f\} \cup\left\{\phi_{j}\right\}_{\jmath=1}^{m-1}\right) .
$$

Hence, by Gram-Schmidt orthogonalization on $\{f\} \cup\left\{\phi_{j}\right\}_{j=1}^{m-1}$, we get the required basis of $S_{k, l}^{q}(\lambda)$.

Next, we prove $A(q)(2)\left(\right.$ ii). For given $f \neq 0 \in S_{k, l}^{q}(\lambda)_{Q(\lambda)}$, take $R>0$ and $\xi \in$ $X_{q}$ so that $a\left(R ; \sigma^{-1} f ; \xi\right) \neq 0$. Let $h(\lambda)$ be the projection of $h_{R, \xi}^{q, q}$ to $S_{k, l}^{q}(\lambda)$. Using $h_{R, \xi}^{q, q} \in S_{k, l_{Q}}^{q}$ and (4.1), we see

$$
h(\lambda)^{\tau}=h\left(\lambda^{\tau}\right)
$$

for $\tau \in \operatorname{Aut}(\boldsymbol{C})$. Let $\left\{f_{1}(=f), \cdots, f_{m}\right\}$ be the orthogonal basis of $A(q)(2)(\mathrm{iii})$.

Writing

$$
h(\lambda)=\sum_{j=1}^{m} \beta_{\jmath} f_{\imath} \quad\left(\beta_{j} \in \boldsymbol{Q}(\lambda)\right),
$$

we have

$$
(f, h(\lambda))=\left(f, h_{R, \xi}^{q, q}\right)=c(f) a\left(R ; \sigma^{-1} f ; \xi\right)
$$

and

$$
(f, h(\lambda))=\left(f, \sum_{j=1}^{m} \beta_{\jmath} f_{\jmath}\right)=\beta_{1}(f, f) .
$$

On the other hand, we get for $\tau \in \operatorname{Aut}(\boldsymbol{C})$,

$$
\left.\left(f^{\tau}, h(\lambda)^{\tau}\right)\right)=\left(f^{\tau}, h_{R, \xi}^{q, q}\right)=c\left(f^{\tau}\right) a\left(R ; \sigma^{-1}\left(f^{\tau}\right) ; \xi\right)
$$

by (4.4) and

$$
\left(f^{\tau}, h\left(\lambda^{\tau}\right)\right)=\left(f^{\tau}, h(\lambda)^{\tau}\right)=\beta_{1}^{\tau}\left(f^{\tau}, f^{\tau}\right)
$$

by (4.5).

Therefore 


$$
\left(\frac{c(f)}{(f, f)}\right)^{\tau}=\frac{\beta_{1}{ }^{\tau}}{a\left(R ; \sigma^{-1}\left(f^{\tau}\right) ; \xi\right)}=\frac{c\left(f^{\tau}\right)}{\left(f^{\tau}, f^{\tau}\right)},
$$

Thus the part (I) is proved.

(II) Suppose that $k>p+q+1$. By $C(q, q)$ and $k \geqq 2 q+2, A(q)$ is valid. For any $R=R^{(p)} \geqq 0$ and $\xi \in X_{p}$, let $h(\lambda)$ be the projection of $h_{R, \xi}^{p, q}$ to $S_{k, l}^{q}(\lambda)$. By $C(p, q),(4.3)$ holds again for this $h(\lambda)$, and by the same argument as in (I), we find a $\beta \in \boldsymbol{Q}(\lambda)$ such that

$$
\begin{gathered}
\beta \frac{(f, f)}{c(f)}=a\left(R ;\left[\sigma^{-1} f\right]_{q}^{p} ; \xi\right), \\
\beta^{\tau} \frac{\left(f^{\tau}, f^{\tau}\right)}{c\left(f^{\tau}\right)}=a\left(R ;\left[\sigma^{-1}\left(f^{\tau}\right)\right]_{q}^{p} ; \xi\right) \quad \text { for all } \quad \tau \in \operatorname{Aut}(\boldsymbol{C}) .
\end{gathered}
$$

Then, from $A(q)(2)$ (ii) and the expression above,

$$
a\left(R ;\left[\sigma^{-1} f\right]_{q}^{p} ; \xi\right)^{\tau}=a\left(R ;\left[\sigma^{-1}\left(f^{\tau}\right)\right]_{q}^{p} ; \xi\right)
$$

for any $R=R^{(p)} \geqq 0, \xi \in X_{p}$ and $\tau \in \operatorname{Aut}(\boldsymbol{C})$. Part (II) is proved.

Remark. $A(q)$ (ii) and (4.1) imply the existence of an orthogonal basis $B_{q}$ of $S_{k, l}^{q}$ such that:

(1) $B_{q}$ is permuted by the action of $\operatorname{Aut}(\boldsymbol{C})$.

(2) Each $f \in B_{q}$ satisfies $f \in S_{k, l_{Q(f)}}$.

Now, we shall show that the condition $C(p, q)$ actually holds when $k$ is sufficiently large.

Proposition 4.2. Let $p \geqq q \geqq 1$ be integers and $k, l \geqq 0$ be even integers such that

Then,

$$
k>p+q+1 \text {. }
$$

(1) $C\left(p^{\prime}, 1\right)$ holds for $1 \leqq p^{\prime} \leqq p$.

(2) Suppose that

$$
C(p, r), C(q, r) \text { and } C(r, r) \text { hold for } 1 \leqq r<q .
$$

Then, $C(p, q)$ holds.

Proof. (1) Let $R^{(p)} \geqq 0$ and $\xi \in X_{p}$ be arbitrary. In this case, $g_{R, \xi}^{p^{\prime}, \xi^{1}}$ and $h_{R, \xi}^{p^{\prime}, \xi^{1}}$ are identified with elliptic modular forms by

and

$$
V_{\boldsymbol{y}}=\boldsymbol{C} y_{1}
$$

Therefore

$$
M_{k, l}^{1}\left(V_{\nu}{ }^{(l)}\right)=M_{k+l, 0}^{1}(C) \cdot y_{1}^{l} .
$$

$$
g_{R, \xi}^{p^{\prime}, 1}-h_{R, \xi}^{p^{\prime}, 1}=a\left(0 ; g_{R, \xi}^{p^{\prime}, 1} ; y_{1}^{l}\right) E_{k+l}^{1} y_{1}^{l},
$$

where $E_{k+l}^{1}: \mathfrak{G}_{1} \rightarrow C$ is the elliptic Eisenstein series, whose Fourier coefficients 
lie in $\boldsymbol{Q}$. Then, by $g_{R, \xi}^{p^{\prime}, 1} \in M_{k, l_{\boldsymbol{Q}}}^{1}$, we see $h_{R, \xi}^{p^{\prime}, 1} \in S_{k, l_{\boldsymbol{Q}}}^{1}$.

(2) By the assumption and Proposition 4.1, we can assume $A(r)$ of Theorem $1, B(q, r)$ and $B(p, r)$ of Theorem 2 for $1 \leqq r<q$, noting that $k>p+r+1 \geqq q+$ $r+1>2 r+1$. In particular by $A(r)(2)$ (iii), for each $r$, there exists an orthogonal basis $B_{r}$ of $S_{k, l}^{r}$ as stated in the Remark above.

By Theorem A of section 3, together with (3.6), (3.7), (3.8), we have

$$
g_{R, \xi}^{p, q}-h_{R, \xi}^{p, q}=\sum_{r=1}^{q-1} \sum_{f \in B_{r}} \frac{c(f)}{(f, f)} a\left(R ;\left[\sigma^{-1} f\right]_{r}^{p} ; \xi\right)[f]_{r}^{q}
$$

for any $R=R^{(p)} \geqq 0$ and $\xi \in X_{p}$.

Since $B_{r}$ is permuted by $\operatorname{Aut}(\boldsymbol{C}), f \in B_{r}$ satisfies $f \in S_{k, l_{Q}(f)}^{r}$, and by $A(r)(2)(\mathrm{ii}), B(q, r), B(p, r)$, we see the right-hand side of (4.6) is invariant

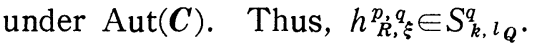

Theorems 1, 2 are proved by induction using Proposition 4.2.

Proof of Theorem 1. Let $q, k, l$ satisfy the assumption. Then, $C(1,1)$ is valid by Proposition 4.2(1).

Let $1 \leqq q^{\prime}<q$ and suppose that

$$
C(m, n) \text { is valid for }(m, n) \text { with } 1 \leqq n \leqq m \leqq q^{\prime} .
$$

Again by Proposition 4.2(1), $C\left(q^{\prime}+1,1\right)$ is valid. By (4.7) and repeated use of Proposition $4.2(2), C\left(q^{\prime}+1, n\right)$ holds for $1 \leqq n \leqq q^{\prime}+1$ (Note that $k \geqq 2 q+2 \geqq$ $\left.2\left(q^{\prime}+1\right)+1\right)$.

Thus we have:

$$
C(m, n) \text { is valid for }(m, n) \text { with } 1 \leqq n \leqq m \leqq q^{\prime}+1,
$$

and finally we obtain $C(q, q)$, which imply Theorem 1 by Proposition 4.1.

Proof of Theorem 2. Let $p, q, k, l$ satisfy the assumption. Then, $C(m, n)$ is valid for $1 \leqq n \leqq m \leqq q$, as seen above. We have $C(p, 1)$, and using Proposition 4.2(2) repeatedly, we get $C(p, q)$ and the assertion of Theorem 2.

\section{REFERENCES}

[1] AndRianov, A.N., Euler products corresponding to Siegel modular forms of genus 2, Russ. Math. Surv. 29 (1974), 45-116 (Engl. transl.).

[2] Böcherer, S., SAtoh, T. and Yamazaki, T., On the Pullback of a Differential Operator and its Application to Vector Valued Eisenstein Series, Commentarii Math. Univ. st. Pauli. 41 (1992) 1-22.

[3] Böcherer, S., Über die Fourier-Jacobi-Entwicklung Siegelscher Eisensteinreihen, Math. Z. 183 (1983), 21-46.

[4] Böcherer, S., Über die Fourier-Jacobi-Entwicklung Siegelscher Eisensteinreihen. II., Math. Z. 189 (1985), 81-110.

[5] GarretT, P. B., Pullbacks of Eisenstein series, applications, Prog. in Math. 46 
(1984), 114-137.

[6] Kurokawa, N., On Siegel Eigenforms, Proc. Japan. Acad. 57A (1981), 47-50.

[7] Mizumoto, S., Poles and residues of standard L-functions attached to Siegel modular forms, Math. Ann. 289 (1991), 589-612.

[8] Siegel, C. L., Einführung in die Theorie der Modulfunktionen n-ten Grades, Math. Ann. 116 (1939), 617-657.

[9] ŻARKovsKajA, N.A., On the connection of the eigenvalues of Hecke operators and the Fourier coefficients of eigenfuncions for Siegel's modular forms of genus n, Math. USSR Sb. 25 (1975), 549-557. (Engl. transl.)

Mihama 2-1-1105,

URAYASU-SHI, CHIBA, 279,

JAPAN 
